\title{
Microwave Assisted Solvent-Free Synthesis of Some Imine Derivatives
}

\author{
Yunus Bekdemir ${ }^{1}$ and Kürşat Efil ${ }^{1,2}$ \\ ${ }^{1}$ Department of Molecular Biology and Genetics, Faculty of Arts and Science, Canik Basarı University, 55080 Samsun, Turkey \\ ${ }^{2}$ Department of Chemistry, Faculty of Arts and Sciences, Ondokuz Mayis University, 55139 Samsun, Turkey
}

Correspondence should be addressed to Yunus Bekdemir; bekdemir@basari.edu.tr

Received 2 January 2014; Revised 23 February 2014; Accepted 24 February 2014; Published 26 March 2014

Academic Editor: Joseph E. Saavedra

Copyright (C) 2014 Y. Bekdemir and K. Efil. This is an open access article distributed under the Creative Commons Attribution License, which permits unrestricted use, distribution, and reproduction in any medium, provided the original work is properly cited.

Some imine derivatives (1a-7d) were synthesized using a rapid and an environmentally friendly method with reaction of aromatic aldehydes (a-d) and aromatic amines (1-7) (in 1:1 molar ratio) in the presence of $\beta$-ethoxyethanol as a wetting reagent ( 2 drops) under solvent-free conditions using microwave heating.

\section{Introduction}

Compounds containing the $-\mathrm{C}=\mathrm{N}-$ (azomethine group) structure are known as Schiff bases, usually synthesized from the condensation of primary amines and active carbonyl groups [1]. The reaction is acid-catalyzed and is generally carried out by refluxing the carbonyl compound and amine, with an azeotroping agent if necessary, and separating the water as formed [2]. Schiff bases are well known for their biological applications as antibacterial, antifungal, anticancer, and antiviral agents; furthermore, they have been used as intermediates in medical substrates and as ligands in complex formation with some metal ions $[1,3]$. The synthesis of imines was firstly reported by Hugo Schiff in 1864 and they have been known since then [4]. The imine compounds have been prepared using molecular sieves $[5,6]$, infrared irradiation [7], $\mathrm{Mg}\left(\mathrm{ClO}_{4}\right)_{2}$ [8], $\mathrm{P}_{2} \mathrm{O}_{5} / \mathrm{SiO}_{2}$ [9], $\mathrm{ZnCl}_{2}$ [10], $\mathrm{CaO}$ under microwave power [11], ethyl lactate as a tunable solvent [12], $\mathrm{K} 10$ clay [13], $\mathrm{TiCl}_{4}$ [14], alumina [15], $\mathrm{CeCl}_{3} \cdot 7 \mathrm{H}_{2} \mathrm{O}$ [16], ultrasound irradiation [17], polymer-supported [18], nanotube $\mathrm{TiO}_{2}$ (in sunlight) [19], and $\mathrm{Ti}(\mathrm{OEt})_{4}[20,21]$.

The present work reveals the comparative aspects of condensation of some aromatic amines with aldehyde derivatives using microwave and conventional methods. The amine and aldehyde compounds as starting materials, $\beta$-ethoxyethanol $(\beta-\mathrm{EE})$ as wetting reagent and microwave power as an effective source of heating are used in this study. The corresponding imine compounds were prepared in high yields and short reaction times using this effective and environment friendly method.

\section{Results and Discussion}

In this work, we synthesized quickly and efficiently a series imine derivatives $(\mathbf{l a}-\mathbf{7} \mathbf{d})$ by condensation of some aromatic amines (1-7) and aldehyde derivatives such as salicylaldehyde (a), p-chlorobenzaldehyde (b), p-methoxybenzaldehyde (c) and cinnamaldehyde (d) under microwave-assisted solventfree conditions using $\beta$-EE as wetting reagent. $\beta$-EE that is a polar molecule quickly absorbs microwaves and therefore heats up and heats around effectively. As a result, $\beta$-EE, which increases the polarity of the reaction medium, has an active role in the heating of the reaction medium by microwaves.

The general reaction was summarized in Scheme 1. In addition, we tested the effect of different microwave power such as $180,360,600,900 \mathrm{~W}$ and detected the microwave power of $180 \mathrm{~W}$ and $360 \mathrm{~W}$ are more appropriate choices for the reaction. Hence, the optimum microwave reaction conditions were determined using 180 and $360 \mathrm{~W}$ microwave power and neat and wetting with $\beta$-EE for compound la. The highest reaction yield (94\%) and shorter reaction time 


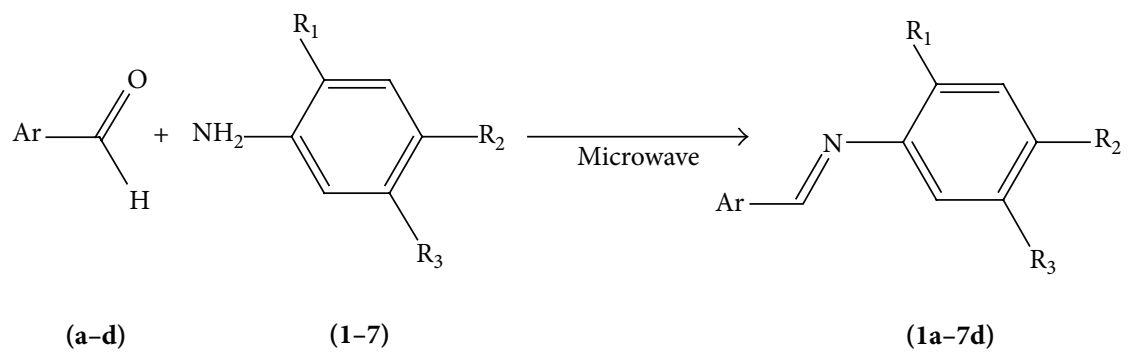

SCHEME 1

TABLE 1: The optimization of microwave conditions for compound 1a.

\begin{tabular}{lccc}
\hline \multicolumn{3}{c}{ Microwave method } \\
Watt & Time (min) & Reaction conditions & Yield (\%) \\
\hline 180 & 5 & Wetting (with $\beta$-EE) & 83 \\
360 & 5 & Wetting (with $\beta$-EE) & 93 \\
360 & 1.5 & Wetting (with $\beta$-EE) & $\mathbf{9 4}$ \\
360 & 5 & Neat & 88 \\
360 & 1.5 & Neat & 81 \\
\hline
\end{tabular}

TABLE 2: The optimization of classical conditions for compound la.

\begin{tabular}{lcc}
\hline Time $(\mathrm{min})$ & $\begin{array}{c}\text { Classical method } \\
\text { Yield (\%) }\end{array}$ & Reaction conditions \\
\hline 30 & 64 & Reflux \\
60 & 73 & Reflux \\
120 & 76 & Reflux \\
240 & 75 & Reflux \\
$1300^{*}$ & 74 & Reflux \\
\hline
\end{tabular}

Procedure: $1 \mathrm{mmol}$ aldehyde $(10 \mathrm{~mL}$ of ethanol) and $1 \mathrm{mmol}$ amine $(10 \mathrm{~mL}$ of ethanol) are mixed a balloon and then boiled under reflux on a hot plate.

*300 min (heated) +1000 min (unheated).

(1.5 min.) were obtained at $360 \mathrm{~W}$ microwave power with $\beta$ EE. In the absence of wetting reagent, the reaction yields are $81 \%$ for 1.5 minutes and $88 \%$ for 5 minutes. It is understood that the reaction yield was increased by wetting reagent that increases the polarity of the reaction medium. The optimization of microwave-assisted conditions was given in Table 1.

In addition, we have synthesized some imine derivatives $(\mathbf{l a}, \mathbf{4 a}, \mathbf{1 b}, \mathbf{4 b}, \mathbf{1 c}, \mathbf{4 c}, \mathbf{1 d}, \mathbf{4 d})$ under classical conditions using a solvent (azeotropic) and traditional heating source (hot plate) for comparison with microwave conditions. Firstly, synthesis of compound la under the classical reaction conditions was tested at various periods of time $(30,60,120,240,1300 \mathrm{~min})$ to determine the optimum reaction time, which was determined as 120 minutes. Information on the optimization of the reaction time of compound la for classic conditions was presented in Table 2.

The compounds $1 \mathbf{a}, \mathbf{4 a}, \mathbf{1 b}, \mathbf{4 b}, \mathbf{1 c}, \mathbf{4 c}, \mathbf{1 d}$, and $\mathbf{4 d}$ were obtained at $76 \%, 73 \%, 63 \%, 79 \%, 67 \%, 73 \%, 67 \%$, and $75 \%$ yields, respectively, in 120 minutes under the classical reaction conditions, whereas in microwave conditions,

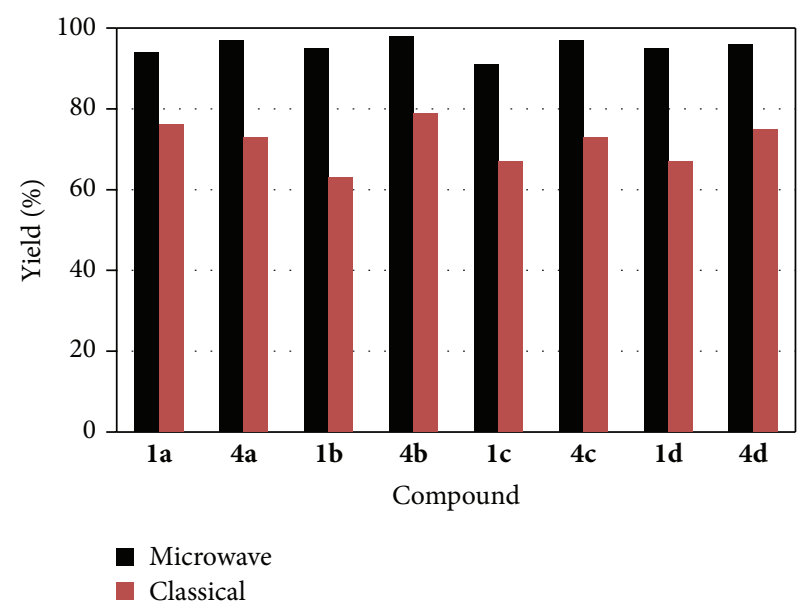

FIGURE 1: Comparison of yields under the microwave and classical conditions.

the corresponding reaction yields were $94 \%, 97 \%, 95 \%, 98 \%$, $91 \%, 97 \%, 95 \%$, and $96 \%$, respectively, in $1.0-1.5$ minutes. All the results such as reactions time, yields, and melting points of the compounds were presented in Table 3 . In addition, the yields of microwave and conventional reaction conditions for some imines were expressed graphically in Figure 1.

\section{Conclusion}

In conclusion, we have developed a simple microwave assisted solvent-free method for the synthesis of imines using a wetting reagent ( $\beta$-ethoxyethanol). The method works well for the reaction of this type amines and aldehydes. Because, we have synthesized imine compounds for good yields and fast reaction times in our method. Our method has some advances such as higher reaction yields, shorter reaction times, and green reaction conditions than classical requirements used of a solvent and conventional heat source (like hot plate) and other some microwave methods used of catalyst and solid supports. In addition, some imines were synthesized for the first time by us with this study.

\section{Experimental}

All of the chemicals were obtained from commercial sources or prepared according to standard methods. Melting points 
TABLE 3: Synthesis of imine derivatives under microwave irradiation.

\begin{tabular}{|c|c|c|c|c|c|c|c|c|c|c|}
\hline \multirow{2}{*}{$\mathrm{N}$} & \multirow{2}{*}{$\mathrm{Ar}$} & \multirow{2}{*}{$\mathrm{R}_{1}$} & \multirow{2}{*}{$\mathrm{R}_{2}$} & \multirow{2}{*}{$\mathrm{R}_{3}$} & \multicolumn{2}{|c|}{ Microwave $^{\mathrm{a}}$} & \multicolumn{2}{|c|}{ Classical $^{\mathrm{b}}$} & \multirow{2}{*}{$\mathrm{m} \cdot \mathrm{p}^{\circ} \mathrm{C}$} & \multirow{2}{*}{ Lit. m.p ${ }^{\circ} \mathrm{C}$} \\
\hline & & & & & Yield \% & $t / \mathrm{min}$ & Yield \% & $t / \mathrm{min}$ & & \\
\hline la & & $\mathrm{OH}$ & $\mathrm{H}$ & $\mathrm{H}$ & 94 & 1.5 & 76 & 120 & 188-190 & $190[22]$ \\
\hline $2 \mathbf{a}$ & & $\mathrm{OH}$ & $\mathrm{H}$ & $\mathrm{Cl}$ & 93 & 1.5 & - & - & 159-162 & $156-156.5$ [23] \\
\hline $3 \mathbf{a}$ & & $\mathrm{OH}$ & $\mathrm{CH}_{3}$ & $\mathrm{H}$ & 93 & 1.5 & - & - & 199-202 & - \\
\hline $4 a$ & & $\mathrm{H}$ & $\mathrm{H}$ & $\mathrm{H}$ & 97 & 1.0 & 73 & 120 & $50-53$ & $50-51$ [24] \\
\hline $5 \mathbf{a}$ & & $\mathrm{H}$ & $\mathrm{Cl}$ & $\mathrm{H}$ & 94 & 1.5 & - & - & 103-105 & $104[12]$ \\
\hline $6 a$ & & $\mathrm{H}$ & $\mathrm{CH}_{3} \mathrm{O}$ & $\mathrm{H}$ & 96 & 1.5 & - & - & $83-85$ & $82-83.5$ [25] \\
\hline $7 \mathbf{a}$ & & $\mathrm{H}$ & $\mathrm{CH}_{3}$ & $\mathrm{H}$ & 97 & 1.5 & - & - & 93-94 & $90-91.5$ [25] \\
\hline lb & & $\mathrm{OH}$ & $\mathrm{H}$ & $\mathrm{H}$ & 95 & 1.5 & 63 & 120 & $116-118$ & 117 [26] \\
\hline $2 b$ & & $\mathrm{OH}$ & $\mathrm{H}$ & $\mathrm{Cl}$ & 92 & 2.0 & - & - & $123-125$ & - \\
\hline $3 b$ & & $\mathrm{OH}$ & $\mathrm{CH}_{3}$ & $\mathrm{H}$ & 91 & 2.0 & - & - & $134-137$ & - \\
\hline $4 \mathrm{~b}$ & & $\mathrm{H}$ & $\mathrm{H}$ & $\mathrm{H}$ & 98 & 1.0 & 79 & 120 & $63-66$ & $64-65[24]$ \\
\hline $5 b$ & & $\mathrm{H}$ & $\mathrm{Cl}$ & $\mathrm{H}$ & 94 & 1.5 & - & - & 110-111 & 110-111 [24] \\
\hline 6b & & $\mathrm{H}$ & $\mathrm{CH}_{3} \mathrm{O}$ & $\mathrm{H}$ & 91 & 3.0 & - & - & $124-126$ & $124-125$ [27] \\
\hline $7 \mathrm{~b}$ & & $\mathrm{H}$ & $\mathrm{CH}_{3}$ & $\mathrm{H}$ & 93 & 1.5 & - & - & $126-128$ & 125 [27] \\
\hline $1 \mathrm{c}$ & & $\mathrm{OH}$ & $\mathrm{H}$ & $\mathrm{H}$ & 91 & 1.5 & 67 & 120 & 90-91 & $91-93$ [22] \\
\hline $2 \mathrm{c}$ & & $\mathrm{OH}$ & $\mathrm{H}$ & $\mathrm{Cl}$ & 95 & 1.0 & - & - & $86-88$ & - \\
\hline $3 c$ & & $\mathrm{OH}$ & $\mathrm{CH}_{3}$ & $\mathrm{H}$ & 93 & 1.5 & - & - & $86-87$ & - \\
\hline $4 \mathrm{c}$ & & $\mathrm{H}$ & $\mathrm{H}$ & $\mathrm{H}$ & 97 & 1.0 & 73 & 120 & $58-60$ & $63-63.5[24]$ \\
\hline $5 \mathrm{c}$ & & $\mathrm{H}$ & $\mathrm{Cl}$ & $\mathrm{H}$ & 97 & 1.5 & - & - & $90-92$ & $92[16]$ \\
\hline 6c & & $\mathrm{H}$ & $\mathrm{CH}_{3} \mathrm{O}$ & $\mathrm{H}$ & 95 & 1.5 & - & - & $147-149$ & $145-146[24]$ \\
\hline $7 \mathrm{c}$ & & $\mathrm{H}$ & $\mathrm{CH}_{3}$ & $\mathrm{H}$ & 91 & 1.5 & - & - & $92-93$ & $91-92$ [24] \\
\hline 1d & & $\mathrm{OH}$ & $\mathrm{H}$ & $\mathrm{H}$ & 95 & 1.5 & 67 & 120 & $89-91$ & $92[22]$ \\
\hline $2 d$ & & $\mathrm{OH}$ & $\mathrm{H}$ & $\mathrm{Cl}$ & 91 & 1.5 & - & - & 91-93 & - \\
\hline $3 d$ & & $\mathrm{OH}$ & $\mathrm{CH}_{3}$ & $\mathrm{H}$ & 94 & 1.5 & - & - & $97-98$ & - \\
\hline $4 d$ & & $\mathrm{H}$ & $\mathrm{H}$ & $\mathrm{H}$ & 96 & 1.0 & 75 & 120 & $108-111$ & $108-109$ [12] \\
\hline $5 d$ & & $\mathrm{H}$ & $\mathrm{Cl}$ & $\mathrm{H}$ & 96 & 1.5 & - & - & 105-107 & $105[12]$ \\
\hline 6d & & $\mathrm{H}$ & $\mathrm{CH}_{3} \mathrm{O}$ & $\mathrm{H}$ & 97 & 1.5 & - & - & $120-121$ & $119-120$ [12] \\
\hline $7 \mathrm{~d}$ & & $\mathrm{H}$ & $\mathrm{CH}_{3}$ & $\mathrm{H}$ & 90 & 1.5 & - & - & $75-77$ & $76-77[28,29]$ \\
\hline
\end{tabular}

${ }^{\mathrm{a}}$ Under solvent-free reaction using microwave.

${ }^{\mathrm{b}}$ Procedure: $1 \mathrm{mmol}$ aldehyde $(10 \mathrm{~mL}$ of ethanol) and $1 \mathrm{mmol}$ amine $(10 \mathrm{~mL}$ of ethanol) are mixed a balloon and then boiled under reflux on a hot plate.

were determined with an Electrothermal 9100 apparatus. The infrared absorption spectrum of the compounds has been recorded in the region at 4000 and $400 \mathrm{~cm}^{-1}$ range using a Bruker Vertex 80/80 v spectrophotometer using $\mathrm{KBr}$ pressed pellet technique at room temperature. The ${ }^{1} \mathrm{H}$ NMR and ${ }^{13} \mathrm{C}$ NMR spectra were recorded using a Bruker $\mathrm{AC}$ $200 \mathrm{NMR} 200 \mathrm{MHz}$ spectrometer in $\mathrm{CDCl}_{3}-d_{1}$ and DMSO$d_{6}$ using TMS as the internal standard. Elemental analyses were conducted at the METU Central Laboratory using a LECO, CHNS-932. All syntheses were carried out in A Bosh HMT 812 C modified microwave oven.

General Procedure for Preparation of 1a-7d. Aldehyde $(1 \mathrm{mmoL})$, amine $(1 \mathrm{mmoL})$ and two drops of $\beta$-ethoxyethanol as a wetting reagent were mixed in a beaker. Then the beaker was placed in the microwave oven and was exposed to microwave irradiation $(360 \mathrm{~W})$. The product obtained by reaction was washed with cold ethanol. Then, it was recrystallized in ethanol. The physical and spectra data of the compounds $\mathbf{3 a}, \mathbf{2 b}, \mathbf{3 b}, \mathbf{2 c}, \mathbf{3 c}, \mathbf{2 d}$, and $\mathbf{3 d}$ are as follows.

$\mathrm{N}$-(Salicylidene)-2-hydroxy-4-methylaniline (3a). M.p. 199202 ${ }^{\circ}$ C. IR: 3033, 2965, 2916, 2858, $1612(\mathrm{C}=\mathrm{N}), 1592,1527$, 1486, 1422, 1278, 1221, 1141, 1113, 1012, 869, 788, 754, 720, 622, $587,556 \mathrm{~cm}^{-1} .{ }^{1} \mathrm{H}$ NMR (DMSO-d $\left.\mathrm{d}_{6}\right): \delta 8.94(\mathrm{~s}, 1 \mathrm{H}, \mathrm{N}=\mathrm{CH})$, $13.90(\mathrm{~s}, 1 \mathrm{H}, \mathrm{OH}), 9.65(\mathrm{~s}, 1 \mathrm{H}, \mathrm{OH}) .{ }^{13} \mathrm{C}$ NMR (DMSO-d 6 ): $\delta 160.54,160.36,150.82,137.56,132.36,132.25,131.92,120.17$, 119.46, 119.14, 118.46, 116.93, 116.48. Elemental Anal. Calcd. for $\mathrm{C}_{14} \mathrm{H}_{13} \mathrm{NO}_{2}$ : C, 73.99; $\mathrm{H}, 5.77 ; \mathrm{N}, 6.16$. Found: C, 72.81; $\mathrm{H}$, $5.64 ; \mathrm{N}, 6.16 \%$.

$N$-(p-Chlorobenzylidene)-2-hydroxy-5-chloroaniline (2b). M.p. $123-125^{\circ}$ C. IR: $3367,3086,3071,3059,2910,2894$, $1626(\mathrm{C}=\mathrm{N}), 1588,1569,1478,1424,1370,1274,1234,1194$, 1154, 1087, 1011, 911, 857, 815, 799 (C-Cl), 697, 658, 608, 548, $533 \mathrm{~cm}^{-1} .{ }^{1} \mathrm{H}$ NMR $\left(\mathrm{CDCl}_{3}\right): \delta 8.66(\mathrm{~s}, 1 \mathrm{H}, \mathrm{N}=\mathrm{CH}), 7.91$ 
$(\mathrm{d}, J=8.4 \mathrm{~Hz}, 2 \mathrm{H}, \mathrm{Ar}), 7.54(\mathrm{~d}, J=8.4 \mathrm{~Hz}, 2 \mathrm{H}, \mathrm{Ar}), 7.34$ (s, $1 \mathrm{H}, \mathrm{Ar}), 7.24$ (d, $J=8.6 \mathrm{~Hz}, 1 \mathrm{H}, \mathrm{Ar}), 7.02(\mathrm{~d}, J=8.6 \mathrm{~Hz}$, $1 \mathrm{H}, \mathrm{Ar}) .{ }^{13} \mathrm{C} \mathrm{NMR}\left(\mathrm{CDCl}_{3}\right): \delta 156.75(\mathrm{~N}=\mathrm{C}), 150.94,138.21$, $135.86,133.87,130.06,129.28,128.77,125.07,116.19,116.16$. Elemental Anal. Calcd. for $\mathrm{C}_{13} \mathrm{H}_{9} \mathrm{Cl}_{2} \mathrm{NO}$ : C, 58,67; $\mathrm{H}, 3,41$; $\mathrm{N}, 5,26$. Found: C, 57.74; H, 3.42; N, 5.26\%.

N-(p-Chlorobenzylidene)-2-hydroxy-4-methylaniline (3b). M.p. $134-137^{\circ}$ C. IR: 3367, 3063, 3049, 3028, 2907, 2857, 1629 $(\mathrm{C}=\mathrm{N}), 1576,1569,1497,1370,1344,1295,1242,1169,1154$, 1080, 1011, 947, 874, 857, 828, 808 (C-Cl), 793, 733, 687, 610, 591, $579 \mathrm{~cm}^{-1} .{ }^{1} \mathrm{H}$ NMR $\left(\mathrm{CDCl}_{3}\right): \delta 8.72(\mathrm{~s}, 1 \mathrm{H}, \mathrm{N}=\mathrm{CH}), 7.92$ (d, $J=8.3 \mathrm{~Hz}, 2 \mathrm{H}, \mathrm{Ar}), 7.53$ (d, $J=8.4 \mathrm{~Hz}, 2 \mathrm{H}, \mathrm{Ar}), 7.29$ (d, $J=8.1 \mathrm{~Hz}, 1 \mathrm{H}, \mathrm{Ar}), 6.92(\mathrm{~s}, 1 \mathrm{H}, \mathrm{Ar}), 6.80(\mathrm{~d}, J=8.0 \mathrm{~Hz}, 1 \mathrm{H}$, Ar), $2.42\left(\mathrm{~s}, 3 \mathrm{H}, \mathrm{CH}_{3}\right) .{ }^{13} \mathrm{C} \mathrm{NMR}\left(\mathrm{CDCl}_{3}\right): \delta 154.09(\mathrm{~N}=\mathrm{C})$, $152.34,139.81,137.40,134.54,132.60,129.72,129.16,120.93$, 115.67, 115.36, 21.48. Elemental Anal. Calcd. for $\mathrm{C}_{14} \mathrm{H}_{12} \mathrm{ClNO}$ C, 68.44; H, 4.92; N, 5.70. Found: C, 67.64; H, 4.89; N, 5.69\%.

N-(p-Methoxybenzylidene)-2-hydroxy-5-chloroaniline (2c). M.p. 86-88 C. IR: 3323, 3017, 2970, 2954, 2927, 2840, 1626 $(\mathrm{C}=\mathrm{N}), 1599,1569,1511,1453,1312,1250,1165,1027,804,650$, $594 \mathrm{~cm}^{-1} .{ }^{1} \mathrm{H}$ NMR $\left(\mathrm{CDCl}_{3}\right): \delta 9.88(\mathrm{~s}, 1 \mathrm{H}, \mathrm{OH}), 8.55(\mathrm{~s}, 1 \mathrm{H}$, $\mathrm{N}=\mathrm{CH}), 7.85$ (d, $J=8.5 \mathrm{~Hz}, 2 \mathrm{H}, \mathrm{Ar}), 7.23$ (s, 1H, Ar), 7.11 (d, $J=8.6 \mathrm{~Hz}, 1 \mathrm{H}, \mathrm{Ar}), 6.99(\mathrm{~d}, J=8.5 \mathrm{~Hz}, 2 \mathrm{H}, \mathrm{Ar}), 6.90$ (d, $J=$ $8.6 \mathrm{~Hz}, 1 \mathrm{H}, \mathrm{Ar}), 3.88\left(\mathrm{~s}, 3 \mathrm{H}, \mathrm{OCH}_{3}\right) .{ }^{13} \mathrm{C} \mathrm{NMR}\left(\mathrm{CDCl}_{3}\right): \delta$ $162.92,157.76(\mathrm{~N}=\mathrm{C}), 150.70,136.69,130.85,128.49,127.81$, 124.91, 116.08, 115.77, 114.33, 55.49. Elemental Anal. Calcd. for $\mathrm{C}_{14} \mathrm{H}_{12} \mathrm{ClNO}_{2}$ : C, 64.25; H, 4.62; N, 5,35. Found: C, 62.92; H, 4.57; N, 5.45\%.

N-(p-Methoxybenzylidene)-2-hydroxy-4-methylaniline (3c). M.p. $86-87^{\circ}$ C. IR: 3344, 3066, 3018, 2974, 2934, 2907, 2840, $1622(\mathrm{C}=\mathrm{N}), 1593,1567,1509,1481,1423,1378,1245,1162,1026$, 969, 909, 861, 835, 809, 761, 666, 617, $559 \mathrm{~cm}^{-1} .{ }^{1} \mathrm{H}$ NMR $\left(\mathrm{CDCl}_{3}\right): \delta 8.59(\mathrm{~s}, 1 \mathrm{H}, \mathrm{N}=\mathrm{CH}), 7.83(\mathrm{~d}, J=8.7 \mathrm{~Hz}, 2 \mathrm{H}, \mathrm{Ar})$, $7.16(\mathrm{~d}, J=8.1 \mathrm{~Hz}, 1 \mathrm{H}, \mathrm{Ar}), 6.97(\mathrm{~d}, J=8.7 \mathrm{~Hz}, 2 \mathrm{H}, \mathrm{Ar}), 6.81$ (s, $1 \mathrm{H}, \mathrm{Ar}), 6.68$ (d, J = 7.8 Hz, $1 \mathrm{H}, \mathrm{Ar}), 3.86$ (s, 3H, $\mathrm{OCH}_{3}$ ), $2.31\left(\mathrm{~s}, 3 \mathrm{H}, \mathrm{CH}_{3}\right) .{ }^{13} \mathrm{C} \mathrm{NMR}\left(\mathrm{CDCl}_{3}\right): \delta 162.52,155.25(\mathrm{~N}=\mathrm{C})$, $152.02,138.71,133.68,130.36,129.13,120.74,115.34,115.26$, 114.30, 55.45, 21.41. Elemental Anal. Calcd. for $\mathrm{C}_{15} \mathrm{H}_{15} \mathrm{NO}_{2}$ : C, 74.67; H, 6.27; N, 5.81. Found: C, 74.63; H, 6.41; N, 6.21\%.

N-(Cinnamylidene)-2-hydroxy-5-chloroaniline (2d). M.p. 91$93^{\circ}$ C. IR: $3344,3064,3013,2964,2911,2881,2839,1621(\mathrm{C}=\mathrm{N})$, $1577,1501,1483,1445,1366,1240,1154,1082,979,837,804,742$, $683,661,593 \mathrm{~cm}^{-1}{ }^{1} \mathrm{H}$ NMR $\left(\mathrm{CDCl}_{3}\right): \delta 8.41(\mathrm{~d}, J=8.2 \mathrm{~Hz}$, $1 \mathrm{H}, \mathrm{N}=\mathrm{CH}), 7.54$ (d, $J=5.2,2 \mathrm{H}, \mathrm{Ar}), 7.43-7.00(\mathrm{~m}, 5 \mathrm{H}, \mathrm{Ar}$ \& $\mathrm{CH}=\mathrm{CH}), 7.29$ (s, $1 \mathrm{H}, \mathrm{Ar}), 7.22(\mathrm{~d}, J=8.1 \mathrm{~Hz}, 1 \mathrm{H}, \mathrm{Ar})$, $6.90(\mathrm{~d}, J=8.6 \mathrm{~Hz}, 1 \mathrm{H}, \mathrm{Ar}) .{ }^{13} \mathrm{C} \mathrm{NMR}\left(\mathrm{CDCl}_{3}\right): \delta 159.36$, $150.95(\mathrm{~N}=\mathrm{C}), 145.23,136.47,135.35,129.99,128.99,128.36$, 128.11, 127.69, 124.94, 115.99, 115.86. Elemental Anal. Calcd, for $\mathrm{C}_{15} \mathrm{H}_{12}$ ClNO: $\mathrm{C}, 69.91 ; \mathrm{H}, 4.69 ; \mathrm{N}, 5.43$. Found: $\mathrm{C}, 68.80 ; \mathrm{H}$, $4.72 ; \mathrm{N}, 5.36 \%$.

N-(Cinnamylidene)-2-hydroxy-4-methylaniline (3d). M.p. 97$98^{\circ}$ C. IR: 3350, 3061, 3012, 2963, 2912, 2880, 2840, 1624 $(\mathrm{C}=\mathrm{N}), 1577,1541,1498,1447,1368,1257,1157,1092,975$, 836, 804, 749, 686, 630, $552 \mathrm{~cm}^{-1}{ }^{1} \mathrm{H}$ NMR $\left(\mathrm{CDCl}_{3}\right): \delta 8.57$ $(\mathrm{d}, J=6.6 \mathrm{~Hz}, 1 \mathrm{H}, \mathrm{N}=\mathrm{CH}), 7.66(\mathrm{dd}, J=6.0,1.9 \mathrm{~Hz}, 2 \mathrm{H}, \mathrm{Ar})$, 7.54-7.12 (m, 5H, Ar \& CH=CH), $7.30(\mathrm{~d}, J=7.9 \mathrm{~Hz}, 1 \mathrm{H}, \mathrm{Ar})$, 6.93 (s, 1H, Ar), 6.80 (d, J = 8.1 Hz, 1H, Ar), 2.43 (s, 3H, Ar). ${ }^{13} \mathrm{C} \mathrm{NMR}\left(\mathrm{CDCl}_{3}\right): \delta 156.85,152.28(\mathrm{~N}=\mathrm{C}), 143.26,139.38$, $135.71,133.20,129.53,128.92,128.73,127.46,120.75,115.48$, 115.04, 21.46. Elemental Anal. Calcd, for $\mathrm{C}_{16} \mathrm{H}_{15} \mathrm{NO}$ : C, 80.98; H, 6.37; N, 5,26. Found: C, 79.22; H, 6.18; N, 5.90\%.

\section{Conflict of Interests}

The authors declare that there is no conflict of interests regarding the publication of this paper.

\section{Acknowledgments}

The authors would like to thank the Middle East Technical University analysis Center (Türkiye) for elemental analyses and Özgür Özdamar (Assistant Professor) and Hasan Saral (Assistant Professor) for the analysis, NMR, and IR of some imine compounds.

\section{References}

[1] A. M. Asiri and S. A. Khan, "Synthesis and anti-bacterial activities of some novel schiff bases derived from aminophenazone," Molecules, vol. 15, no. 10, pp. 6850-6858, 2010.

[2] R. W. Layer, "The chemistry of imines," Chemical Reviews, vol. 63, pp. 489-510, 1963.

[3] H.-K. Fun, R. Kia, A. M. Vijesh, and A. M. Isloor, "5-Diethylamino-2-[(E)-(4-methyl-3-nitrophenyl)iminomethyl]phenol: a redetermination," Acta Crystallographica E: Structure Reports Online, vol. 65, no. 2, pp. o349-o350, 2009.

[4] H. Schiff, "Sur quelques dérivés phéniques des aldéhydes," Annali Di Chimica, vol. 131, p. 118, 1864.

[5] K. Taguchi and F. H. Westheimer, "Catalysis by molecular sieves in the preparation of ketimines and enamines," Journal of Organic Chemistry, vol. 36, no. 11, pp. 1570-1572, 1971.

[6] M. E. Kuehne, "The application of enamines to a new synthesis of $\beta$-ketonitriles," Journal of the American Chemical Society, vol. 81, no. 20, pp. 5400-5404, 1959.

[7] M. Á. Vázquez, M. Landa, L. Reyes, R. Miranda, J. Tamariz, and F. Delgado, "Infrared irradiation: effective promoter in the formation of N-benzylideneanilines in the absence of solvent," Synthetic Communications, vol. 34, no. 15, pp. 2705-2718, 2004.

[8] A. K. Chakraborti, S. Bhagat, and S. Rudrawar, "Magnesium perchlorate as an efficient catalyst for the synthesis of imines and phenylhydrazones," Tetrahedron Letters, vol. 45, no. 41, pp. 7641-7644, 2004.

[9] H. Naeimi, H. Sharghi, F. Salimi, and K. Rabiei, "Facile and efficient method for preparation of Schiff bases catalyzed by $\mathrm{P}_{2} \mathrm{O}_{5} / \mathrm{SiO}_{2}$ under free Solvent conditions," Heteroatom Chemistry, vol. 19, no. 1, pp. 43-47, 2008.

[10] J. H. Billman and K. M. Tai, "Reduction of Schiff bases. II: benzhydrylamines and structurally related compounds," Journal of Organic Chemistry, vol. 23, no. 4, pp. 535-539, 1958.

[11] M. Gopalakrishnan, P. Sureshkumar, V. Kanagarajan, and J. Thanusu, "New environmentally-friendly solvent-free synthesis of imines using calcium oxide under microwave irradiation," Research on Chemical Intermediates, vol. 33, no. 6, pp. 541-548, 2007. 
[12] J. S. Bennett, K. L. Charles, M. R. Miner et al., "Ethyl lactate as a tunable solvent for the synthesis of aryl aldimines," Green Chemistry, vol. 11, no. 2, pp. 166-168, 2009.

[13] R. S. Varma, R. Dahiya, and S. Kumar, "Clay catalyzed synthesis of imines and enamines under solvent-free conditions using microwave irradiation," Tetrahedron Letters, vol. 38, no. 12, pp. 2039-2042, 1997.

[14] W. A. White and H. Weingarten, "A versatile new enamine synthesis," Journal of Organic Chemistry, vol. 32, no. 1, pp. 213214, 1967.

[15] F. Texier-Boullet, "A simple, convenient and mild synthesis of imines on alumina surface without solvent," Synthesis, vol. 1985, no. 6-7, pp. 679-681, 1985

[16] L. Ravishankar, S. A. Patwe, N. Gosarani, and A. Roy, "Cerium(III)-catalyzed synthesis of schiff bases: a green approach," Synthetic Communications, vol. 40, no. 21, pp. 31773180, 2010.

[17] K. P. Guzen, A. S. Guarezemini, A. T. G. Órfão, R. Cella, C. M. P. Pereira, and H. A. Stefani, "Eco-friendly synthesis of imines by ultrasound irradiation," Tetrahedron Letters, vol. 48, no. 10, pp. 1845-1848, 2007.

[18] R. Annunziata, M. Benaglia, M. Cinquini, and F. Cozzi, "Poly(ethylene glycol)-supported 4-alkylthio-substituted aniline: a useful starting material for the soluble polymer-supported synthesis of imines and 1,2,3,4-tetrahydroquinolines," European Journal of Organic Chemistry, no. 7, pp. 1184-1190, 2002.

[19] M. Hosseini-Sarvari, "Nano-tube $\mathrm{TiO}_{2}$ as a new catalyst for eco-friendly synthesis of imines in sunlight," Chinese Chemical Letters, vol. 22, no. 5, pp. 547-550, 2011.

[20] G. Dutheuil, S. Couve-Bonnaire, and X. Pannecoucke, "Diastereomeric fluoroolefins as peptide bond mimics prepared by asymmetric reductive amination of $\alpha$-fluoroenones," Angewandte Chemie: International Edition, vol. 46, no. 8, pp. 12901292, 2007.

[21] J. F. Collados, E. Toledano, D. Guijarro, and M. Yus, "Microwave-assisted solvent-free synthesis of enantiomerically pure $\mathrm{N}$-(tert-Butylsulfinyl)imines," Journal of Organic Chemistry, vol. 77, Article ID 300919, pp. 5744-5750, 2012.

[22] E. Tauer and K. H. Grellmann, "Photochemical and thermal reactions of aromatic Schiff bases," Journal of Organic Chemistry, vol. 46, no. 21, pp. 4252-4258, 1981.

[23] R. J. Argauer and C. E. White, "Effect of substituent groups on fluorescence of metal chelates," Analytical Chemistry, vol. 36, no. 11, pp. 2141-2148, 1964.

[24] J. D. Margerum and J. A. Sousa, "Spectroscopic studies of substituted benzalanilines," Applied Spectroscopy, vol. 19, pp. 9197, 1965.

[25] J. E. Kuder, H. W. Gibson, and D. Wychick, "Electrochemical characterization of salicylaldehyde anils," Journal of Organic Chemistry, vol. 40, no. 7, pp. 875-879, 1975.

[26] F. F. Stephens and J. D. Bower, "The preparation of benziminazoles and benzoxazoles from Schiff's bases. Part I," Journal of the Chemical Society, pp. 2971-2972, 1949.

[27] J. Schmeyers, F. Toda, J. Boy, and G. Kaupp, "Quantitative solidsolid synthesis of azomethines," Journal of the Chemical Society: Perkin Transactions 2, no. 4, pp. 989-993, 1998.

[28] W. Xu, S. Zhang, S. Yang et al., "Asymmetric synthesis of $\alpha$ aminophosphonates using the inexpensive chiral catalyst $1,1^{\prime}$ binaphthol phosphate," Molecules, vol. 15, no. 8, pp. 5782-5796, 2010.
[29] Y. M. S. A. Al-Kahraman, H. M. F. Madkour, D. Ali, and M. Yasinzai, "Antileishmanial, antimicrobial and antifungal activities of some new aryl azomethines," Molecules, vol. 15, no. 2, pp. 660-671, 2010. 

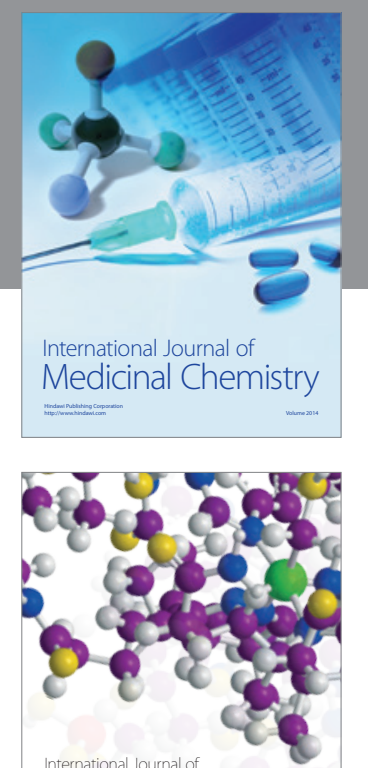

\section{Carbohydrate} Chemistry

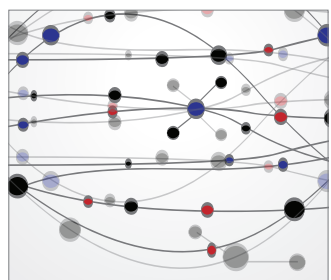

The Scientific World Journal
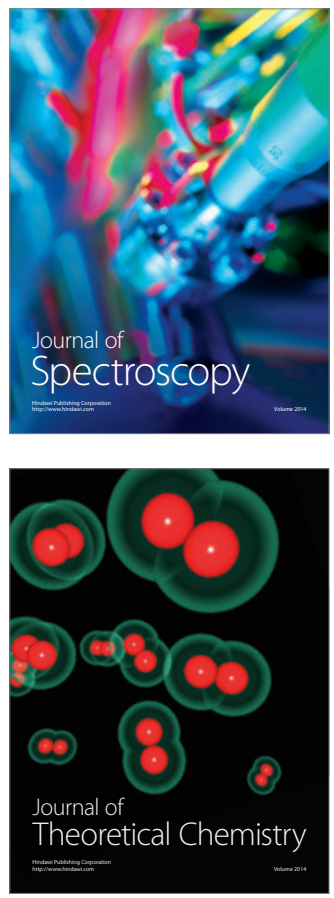
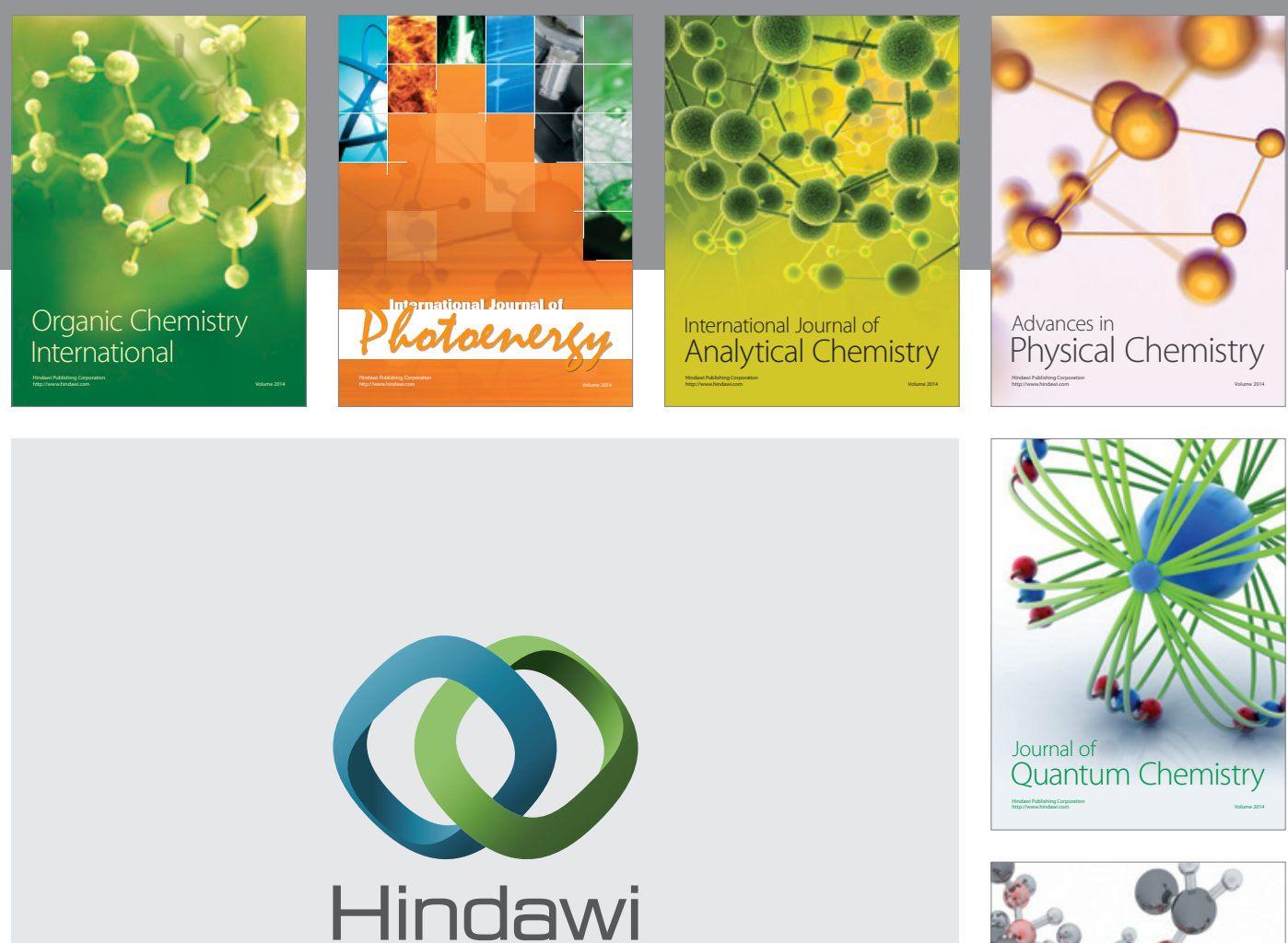

Submit your manuscripts at

http://www.hindawi.com

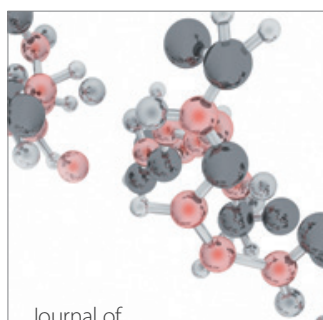

Analytical Methods

in Chemistry

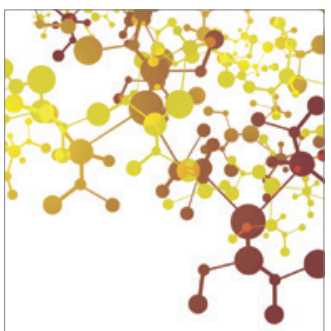

Journal of

Applied Chemistry

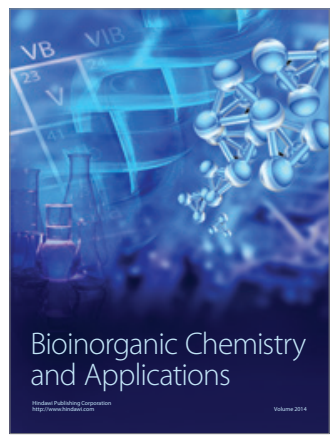

Inorganic Chemistry
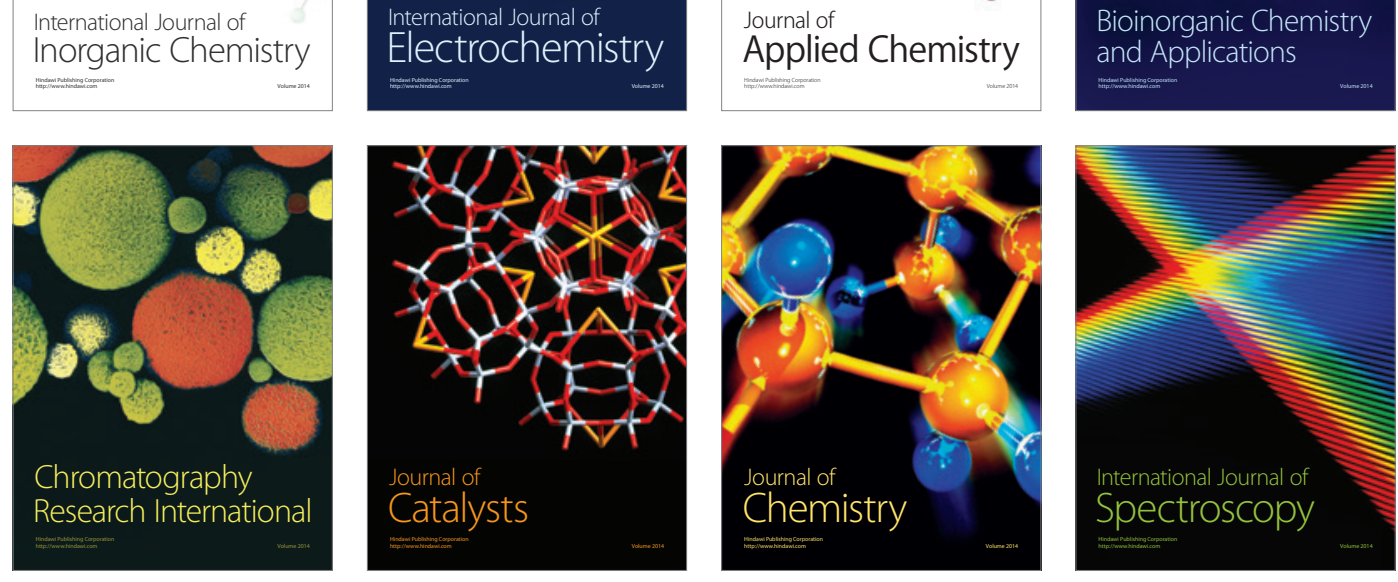